\title{
TITLE:
}

\section{Independent Spanning Trees of Chordal Rings}

AUTHOR(S):

Iwasaki, Yukihiro; Kajiwara, Yuka; Obokata, Koji; Igarashi, Yoshihide

\section{CITATION:}

Iwasaki, Yukihiro ...[et al]. Independent Spanning Trees of Chordal Rings. 数理解析研究所 講究録 1997, 992: 82-89

ISSUE DATE:

1997-05

URL:

http://hdl.handle.net/2433/61153

RIGHT: 


\title{
Independent Spanning Trees of Chordal Rings
}

\author{
Yukihiro Iwasaki Yuka Kajiwara Koji Obokata Yoshihide Igarashi \\ 岩崎 至宏梶原由香尘保方次五十嵐善英 \\ Department of Computer Science, Gunma University \\ E-mail: igarashi@comp.cs.gunma-u.ac.jp
}

\section{Introduction}

A chordal ring, denoted by $C R(N, d)$, is a graph $G=(V, E)$, where $V=\{0,1, \cdots, N-1\}, E=$ $\left\{(u, v) \mid[v-u]_{N}=1\right.$ or $\left.d\right\}, 2 \leq d \leq N / 2$, and $[r]_{N}$ denotes $r$ modulo $N$. If $2 \leq d<N / 2$ then $C R(N, d)$ is 4-regular, and if $4 \leq 2 d=N$ then $C R(N, d)$ is 3-regular. Examples of chordal rings are shown in Figure 1. Chordal rings have a number of advantages as interconnection networks. For any pair of $N$ and $d$ satisfying $2 \leq d \leq N / 2$, chordal ring $C R(N, d)$ exists. They are regular and with fixed degrees ( 3 or 4$)$. It can be easily extended or reduced. For example, in order to extend $C R(N, d)$ to $C R(N+1, d)$, we need only $O(d)$ connection changes.

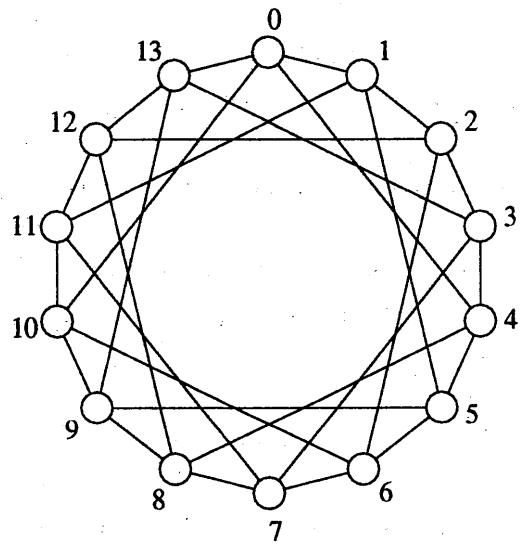

$C R(14,4)$

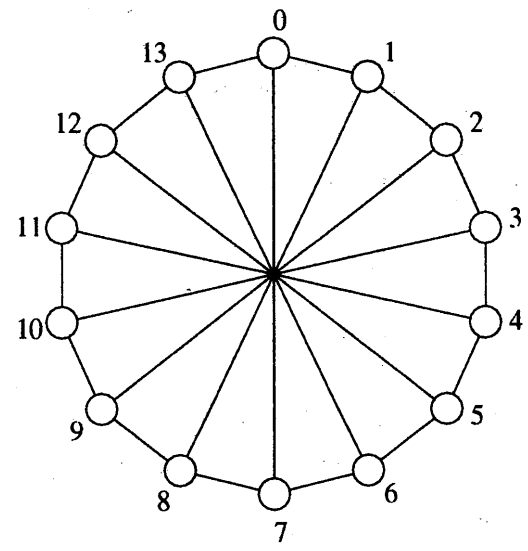

$C R(14,7)$

Figure 1: Chordal rings

A set of paths connecting a pair of vertices in a graph are said to be internally disjoint if and only if any pair of the set have no common vertices and no common edges except for their extreme vertices. Two spanning trees of a graph $G=(V, E)$ are said to be independent if they are rooted at the same vertex, say $r$, and for each vertex $v$ in $V$, the two paths from $r$ to $v$, one path in each tree are internally disjoint. A set of spanning trees of a graph are said to be independent if they are pairwise independent. A graph $G$ is called an $n$-channel graph at vertex $r$ if there are $n$ independent spanning trees rooted at $r$ of $G$. If $G$ is an $n$-channel graph at every vertex, $G$ is called an $n$-channel graph. For example, the $n$-dimensional hypercube ( $n$-cube for short) is an $n$-channel graph. Three independent spanning trees rooted at the same vertex of the 3-cube are shown in Figure 2, where bold lines denote edges of independent spanning trees.

Itai and Rodeh [9] gave a linear time algorithm for finding two independent spanning trees in a biconnected graph. Cheriyan and Maheshwari [6] showed how to find three independent spanning trees of any 3-connected graph $G=(V, E)$ in $O(|V||E|)$ time. Zehavi and Itai [12] showed also that for any 3-connected graph $G$ and for any vertex $r$ of $G$ there are three independent spanning trees rooted at $r$. They conjectured [10][12] that any $k$-connected graph has $k$ independent spanning trees 

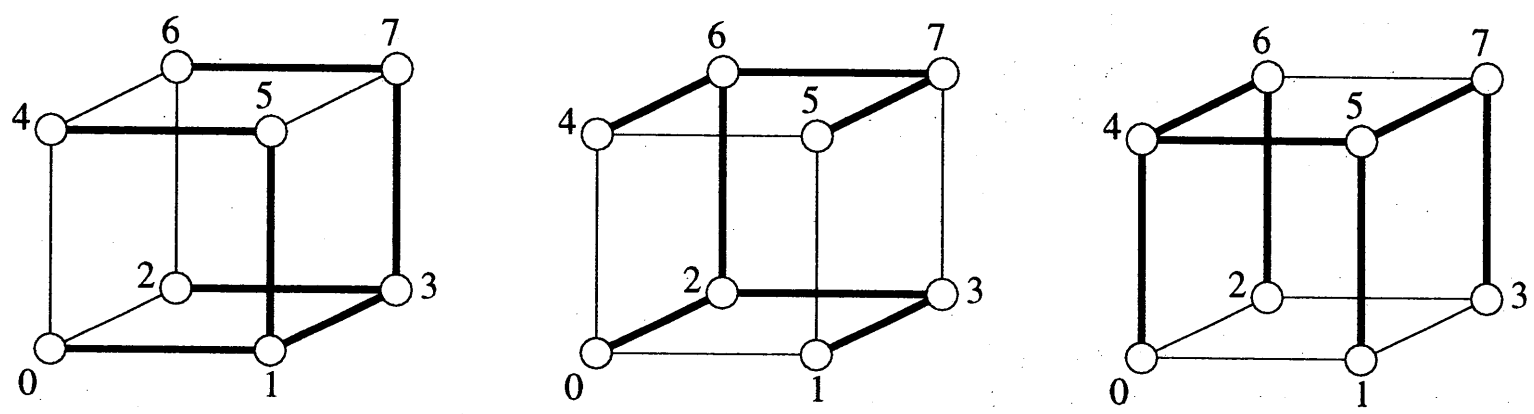

Figure 2: Independent spanning trees rooted at 0 of the 3 -cube

rooted at an arbitrary vertex $r$. Recently Huck has proved that for any $k$-connected planar graph with $k=4$ or $k=5$ (i.e., for any planar graph since any $k$-connected graph with $k \geq 6$ is nonplanar) there are $k$ independent spanning trees rooted at any vertex [7][8][5]. For arbitrary $k$-connected graphs with $k \geq 4$, the conjecture is still open. It has been also shown that if $G_{1}$ is an $n_{1}$-channel graph and $G_{2}$ is an $n_{2}$-channel graph, then the product graph of $G_{1}$ and $G_{2}$, denoted by $G_{1} \times G_{2}$, is an $\left(n_{1}+n_{2}\right)$-channel graph [11]. From this fact it is immediate that the $n$-cube, an $n$-dimensional mesh and an $n$-dimensional torus are an $n$-channel graph, an $n$-channel graph, and a $2 n$-channel graph, respectively.

Broadcasting in a distributed system is the message dissemination from a source processor to every other processor in the system. It has been shown that broadcasting along independent spanning trees are efficient and reliable [2][3][4][9]. In fact, if $G$ is an $n$-channel graph and the source vertex is faultless, then there exists a broadcasting scheme that can tolerate up to $n-1$ faults of the crash type and up to $\lfloor(n-1) / 2\rfloor$ faults of the Byzantine type. All transmissions by such a broadcasting scheme contribute to the majority voting to obtain the correct message, and its communication complexity is optimal to tolerate up to $\lfloor(n-1) / 2\rfloor$ faults of the Byzantine type [2][3][4].

In general it is hard to construct $n$ independent spanning trees rooted at the same vertex of a given $n$-connected graph. If $N$ is even and $d=2$ then $C R(N, d)$ is planar. If $d=2$ and $N$ is an odd integer greater than 4 , or if $3 \leq d \leq N / 2$, then $C R(N, d)$ is nonplanar. In this paper we show how to construct 4 independent spanning trees of $C R(N, d)$ for $2 \leq d<N / 2$ and how to construct 3 independent spanning trees of $C R(N, d)$ for $2 \leq d=N / 2$. From the construction we can say that $C R(N, d)$ is a 4-channel graph for $2 \leq d<N / 2$, and a 3-channel graph for $2 \leq d=N / 2$.

\section{Construction of Independent Spanning Trees}

Chordal rings are symmetric with respect to vertices. Hence, without loss of generality we may only consider independent spanning trees rooted at vertex 0 of each chordal ring. We first assume $2 \leq d<N / 2$.

procedure construct $1(N, d)$;

$(* 2 \leq d<N / 2 *)$

begin

(1) for $i:=0$ to $d-2$ do

connect vertex $i$ with vertex $i+1$;

(2) for $i:=1$ to $d-1$ do

connect vertex $i$ with vertex $[i-d]_{N}$;

(3) for $i:=1$ to $d-1$ do begin 
$x:=i$

while $x \leq N-2 d$ do begin

connect vertex $x$ with vertex $x+d$;

$x:=x+d$

end

end;

(4) $x:=d-1$;

while $x \leq N-d-1$ do begin

connect vertex $x$ with vertex $x+1$;

$x:=x+d$

end

end

Let $T_{1}(N, d)$ be a graph with the vertex set $\{0,1, \cdots, N-1\}$ and the edge set specified by procedure construct $1(N, d)$. We show $T_{1}(14,4)$ in Figure 3 , where the edges of $T_{1}(N, d)$ are indicated by the bold lines.

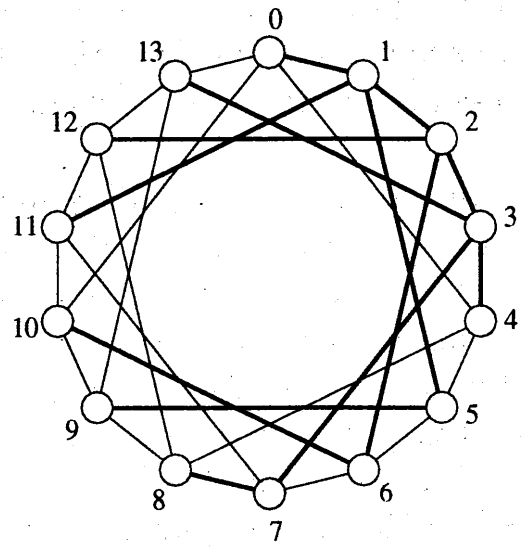

Figure 3: A graph constructed by procedure construct $1, T_{1}(14,4)$

We use notation $x \stackrel{+i}{\longrightarrow} y$ to denote the path of length 1 from vertex $x$ to vertex $y$ such that $[y-x]_{N}=i$. Notation $x \stackrel{-i}{\longrightarrow} y$ denotes the path of length 1 from vertex $x$ to vertex $y$ such that $[y-x]_{N}=-i$. The reflexive and transitive closures of $\stackrel{+i}{\longrightarrow}$ and of $\stackrel{-i}{\longrightarrow}$ are denoted by $\stackrel{+i}{\longrightarrow}$ and $\stackrel{-i}{\longrightarrow}$, respectively.

Lemma $1 T_{1}(N, d)$ is a spanning tree of $C R(N, d)$.

Proof: The vertex set of $T_{1}(N, d)$ is $\{0,1, \cdots, N-1\}$, and any edge $(x, y)$ of $T_{1}(N, d)$ satisfies $[x-y]_{N}=1,-1, d$, or $-d$. Hence, $T_{1}(N, d)$ is a spanning subgraph of $C R(N, d)$. The sets of edges constructed by (1), (2), (3) and (4) of procedure construct $1(N, d)$ consist of $d-1$ edges, $d-1$ edges, $N-2 d-(\lfloor N / d\rfloor-2)$ edges and $\lfloor N / d\rfloor-1$ edges, respectively. These sets of edges are disjoint. Hence, the number of edges of $T_{1}(N, d)$ is $2(d-1)+(N-2 d-\lfloor N / d\rfloor+2)+(\lfloor N / d\rfloor-1)=N-1$.

Let $x$ be a vertex of $C R(N, d)$. If $1 \leq x \leq d-1$ then there is a path $0 \stackrel{+1}{\Longrightarrow} x$ in $T_{1}(N, d)$. If $d \leq x \leq N-d$ and $[x]_{d}=0$ then there is a path $0 \stackrel{+1}{\Longrightarrow} d-1 \stackrel{+d}{\Longrightarrow} x-1 \stackrel{+1}{\Longrightarrow} x$. If $d \leq x \leq N-d$ and $[x]_{d}=y$ for some $0<y<d$ then there is a path $0 \stackrel{+1}{\Longrightarrow} y \stackrel{+d}{\Longrightarrow} x$. If $N-d<x \leq N-1$ then there is a path $0 \stackrel{+1}{\Longrightarrow}[x+d]_{N} \stackrel{-d}{\longrightarrow} x$. Hence, for any vertex $x$ of $C R(N, d)$, there is a path from 0 to $x$ in $T_{1}(N, d)$. Therefore, $T_{1}(N, d)$ is a spanning tree of $C R(N, d)$. 
procedure construct $2(N, d)$;

$\left(* 2 \leq d<N / 2^{*}\right)$

begin

(1) $\quad x:=0$

while $x \leq N-2 d$ do begin

connect vertex $x$ with vertex $x+d$;

$x:=x+d$

end

(2) for $i:=d$ to $N-d-1$ do

if $[i+1]_{d} \neq 0$ then

connect vertex $i$ with vertex $i+1$;

(3) for $i:=d$ downto 2 do

connect vertex $i$ with vertex $i-1$;

(4) for $i:=N-2 d+1$ to $N-d-1$ do

connect vertex $i$ with vertex $i+d$;

end

Let $T_{2}(N, d)$ be a graph with the vertex set $\{0,1, \cdots, N-1\}$ and the edge set specified by procedure construct $2(N, d)$. We show $T_{2}(14,4)$ in Figure 4 , where the edges of $T_{2}(14,4)$ are indicated by the bold lines.

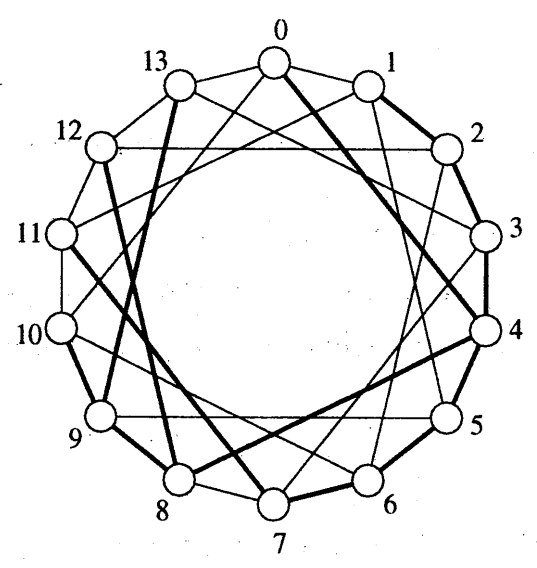

Figure 4: A graph constructed by procedure construct:2, $T_{2}(14,4)$

Lemma $2 T_{2}(N, d)$ is a spanning tree of $C R(N, d)$.

Proof: The vertex set of $T_{2}(N, d)$ is $\{0,1, \cdots, N-1\}$, and any edge $(x, y)$ of $T_{2}(N, d)$ satisfies $[x-y]_{N}=1,-1, d$, or $-d$. Hence, $T_{2}(N, d)$ is a spanning subgraph of $C R(N, d)$. The sets of edges constructed by (1), (2), (3) and (4) of procedure construct $2(N, d)$ are disjoint, and these sets consist of $\lfloor N / d\rfloor-1$ edges, $N-2 d-\lfloor N / d\rfloor+2$ edges, $d-1$ edges and $d-1$ edges, respectively. Hence, the number of edges of $T_{2}(N, d)$ is $\lfloor N / d\rfloor-1+N-2 d-\lfloor N / d\rfloor+2+d-1+d-1=N-1$.

Let $x$ be a vertex of $C R(N, d)$. If $1 \leq x \leq d-1$ then there is a path $0 \stackrel{+d}{\longrightarrow} d \stackrel{-1}{\Longrightarrow} x$ in $T_{2}(N, d)$. If $d \leq x \leq N-d$ and $[x]_{d}=0$ then there is a path $0 \stackrel{+d}{\Longrightarrow} x$. If $d \leq x \leq N-d$ and $[x]_{d}=y$ for some $0<y<d$ then there is a path $0 \stackrel{+d}{\Longrightarrow} x-y \stackrel{+1}{\Longrightarrow} x$. If $N-d<x \leq N-1$ and $[x]_{d}=y$ for some $0 \leq y<d$ then there is a path $0 \stackrel{+d}{\Longrightarrow} x-d-y \stackrel{+1}{\Longrightarrow} x-d \stackrel{+d}{\longrightarrow} x$. Hence, for any vertex $x$ of $C R(N, d)$, there is a path from 0 to $x$ in $T_{2}(N, d)$. Therefore, $T_{2}(N, d)$ is a spanning tree of $C R(N, d)$. 
Symmetrically we can define $T_{3}(N, d)$ and $T_{4}(N, d)$ as follows: Let $T_{3}(N, d)$ be a spanning tree of $C R(N, d)$ obtained from $T_{1}(N, d)$ by changing the name of each vertex $x$ to $[N-x]_{N}$. Let $T_{4}(N, d)$ be a spanning tree of $C R(N, d)$ obtained from $T_{2}(N, d)$ by changing the name of each vertex $x$ to $[N-x]_{N}$. Examples of $T_{3}(N, d)$ and $T_{4}(N, d)$ are shown in Figure 5 , where the bold lines are the edges of the spanning trees.
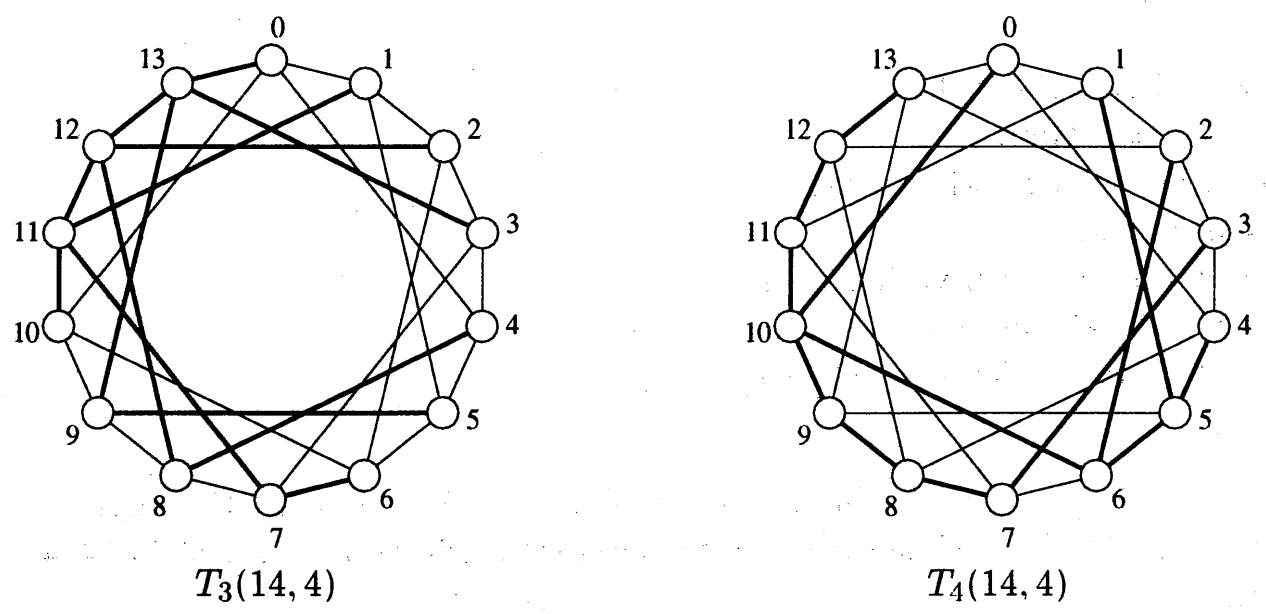

Figure 5: Spanning trees $T_{3}(14,4)$ and $T_{4}(14,4)$

Lemma $3 T_{1}(N, d)$ and $T_{2}(N, d)$ are independent spanning trees rooted at 0 of $C R(N, d)$.

Proof: We consider the paths from 0 to $x$ in $T_{1}(N, d)$ and in $T_{2}(N, d)$. When $1 \leq x \leq d-1$, these paths are $0 \stackrel{+1}{\Longrightarrow} x$ and $0 \stackrel{+d}{\longrightarrow} d \stackrel{-1}{\Longrightarrow} x$. Any internal vertex $a$ of $0 \stackrel{+1}{\Longrightarrow} x$ satisfies $0<a<x$, whereas any internal vertex $b$ of $0 \stackrel{+d}{\longrightarrow} d \stackrel{-1}{\Longrightarrow} x$ satisfies $x<b \leq d$. Hence, these two paths are internally disjoint.

We next assume that $d \leq x \leq N-d$. If $[x]_{d}=0$ then the paths from 0 to $x$ in $T_{1}(N, d)$ and in $T_{2}(N, d)$ are $0 \stackrel{+1}{\Longrightarrow} d-1 \stackrel{+d}{\Longrightarrow} x-1 \stackrel{+1}{\longrightarrow} x$ and $0 \stackrel{+d}{\Longrightarrow} x$, respectively. Any internal vertex $a$ of the former path satisfies $0<[a]_{d} \leq d-1$, whereas any internal vertex $b$ of the latter path satisfies $[b]_{d}=0$. Hence, these two paths are internally disjoint. If $[x]_{d}=y$ for some $0<y<d$ then the paths from 0 to $x$ in $T_{1}(N, d)$ and in $T_{2}(N, d)$ are $0 \stackrel{+1}{\Longrightarrow} y \stackrel{+d}{\Longrightarrow} x$ and $0 \stackrel{+d}{\Longrightarrow} d \stackrel{+d}{\Longrightarrow} x-y \stackrel{+1}{\Longrightarrow} x$, respectively. Any internal vertex $a$ of the subpath $y \stackrel{+d}{\Longrightarrow} x$ satisfies $[a]_{d}=y$, whereas any internal vertex $b$ of the subpath $d \stackrel{+d}{\Longrightarrow} x-y \stackrel{+1}{\Longrightarrow} x$ satisfies $0 \leq[b]_{d}<y$. Hence, these two paths from 0 to $x$ are internally disjoint.

We finally assume that $N-d<x \leq N-1$ and $[x]_{d}=y$ for some $0 \leq y<d$. The paths from 0 to $x$ in $T_{1}(N, d)$ and in $T_{2}(N, d)$ are $0 \stackrel{+1}{\Longrightarrow}[x+d]_{N} \stackrel{-d}{\longrightarrow} x$ and $0 \stackrel{+d}{\longrightarrow} d \stackrel{+d}{\Longrightarrow} x-d-y \stackrel{+1}{\Longrightarrow} x-d \stackrel{+d}{\longrightarrow} x$, respectively. Any internal vertex of the former path is less than $d$, whereas any internal vertex of the latter path is not less than $d$. Hence, these two paths are internally disjoint. Therefore, $T_{1}(N, d)$ and $T_{2}(N, d)$ are independent spanning trees rooted at 0 of $C R(N, d)$.

Symmetrically, we have the next lemma.

Lemma $4 T_{3}(N, d)$ and $T_{4}(N, d)$ are independent spanning trees rooted at 0 of $C R(N, d)$.

Lemma $5 T_{1}(N, d)$ and $T_{3}(N, d)$ are independent spanning trees rooted at 0 of $C R(N, d)$. 
Proof: We first assume that $1 \leq x \leq d-1$. The paths from 0 to $x$ in $T_{1}(N, d)$ and in $T_{3}(N, d)$ are $0 \stackrel{+1}{\Longrightarrow} x$ and $0 \stackrel{-1}{\Longrightarrow}[x-d]_{N} \stackrel{+d}{\longrightarrow} x$, respectively. For any internal vertex $a$ of the former path and any internal vertex $b$ of the latter path, $0<a<x$ and $x<N-d<b$. Hence, these two paths are internally disjoint.

We next assume that $d \leq x \leq N-d$. If $[x]_{d}=0$ then the path from 0 to $x$ in $T_{1}(N, d)$ is $0 \stackrel{+1}{\Longrightarrow} d-1 \stackrel{+d}{\Longrightarrow} x-1 \stackrel{+1}{\longrightarrow} x$, and if $[x]_{d}=y$ for some $0<y<d$ then the path from 0 to $x$ is $0 \stackrel{+1}{\Longrightarrow} y \stackrel{+d}{\Longrightarrow} x$. For each case any internal vertex $a$ of the path satisfies $0<a<x$. If $[N-x]_{d}=0$ then the path from 0 to $x$ in $T_{3}(N, d)$ is $0 \stackrel{-1}{\Longrightarrow} N-d+1 \stackrel{-d}{\Longrightarrow} x+1 \stackrel{-1}{\longrightarrow} x$, and if $[N-x]_{d}=y$ for some $0<y<d$ then the path from 0 to $x$ in $T_{3}(N, d)$ is $0 \stackrel{-1}{\Longrightarrow} N-y \stackrel{-d}{\Longrightarrow} x$. For each case any internal vertex $b$ of the path satisfies $x<b$. Hence, the paths from 0 to $x$ in $T_{1}(N, d)$ and in $T_{3}(N, d)$ are internally disjoint.

Finally we assume that $N-d<x \leq N-1$. The paths from 0 to $x$ in $T_{1}(N, d)$ and in $T_{3}(N, d)$ are $0 \stackrel{+1}{\Longrightarrow}[x+d]_{N} \stackrel{-d}{\longrightarrow} x$ and $0 \stackrel{-1}{\Longrightarrow} x$, respectively. For any internal vertex $a$ of the former path $0<a<d$, and for any internal vertex $b$ of the latter path $x<b$. Hence, these two paths are internally disjoint. Therefore, $T_{1}(N, d)$ and $T_{3}(N, d)$ are independent spanning trees rooted at 0 of $C R(N, d)$.

Lemma $6 T_{2}(N, d)$ and $T_{4}(N, d)$ are independent spanning trees rooted at 0 of $C R(N, d)$.

Proof: We first assume that $1 \leq x \leq d-1$. Let $[N-x]_{d}=y$ for some $0 \leq y<d$. Then the paths from 0 to $x$ in $T_{2}(N, d)$ and in $T_{4}(N, d)$ are $0 \stackrel{+d}{\longrightarrow} d \stackrel{-1}{\Longrightarrow} x$ and $0 \stackrel{-d}{\Longrightarrow} x+d+y \stackrel{-1}{\Longrightarrow} x+d \stackrel{-d}{\longrightarrow} x$, respectively. These two paths are internally disjoint.

We next assume that $d \leq x \leq N-d$. If $[x]_{d}=0$ then the path from 0 to $x$ in $T_{2}(N, d)$ is $0 \stackrel{+d}{\Longrightarrow} x$, and if $[x]_{d}=y$ for some $0<y<d$ then the path from 0 to $x$ in $T_{2}(N, d)$ is $0 \stackrel{+d}{\Longrightarrow} x-y \stackrel{+b}{\Longrightarrow} x$. In each case any internal vertex $a$ of the path satisfies $0<a<x$. If $[N-x]_{d}=0$ then the path from 0 to $x$ in $T_{4}(N, d)$ is $0 \stackrel{-d}{\Longrightarrow} x$, and if $[N-x]_{d}=y$ for some $0<y<d$ then the path from 0 to $x$ in $T_{4}(N, d)$ is $0 \stackrel{-d}{\Longrightarrow} x+y \stackrel{-1}{\Longrightarrow} x$. In each case any internal vertex $b$ of the path satisfies $x<b$. Hence, for any case the paths from 0 to $x$ in $T_{2}(N, d)$ and in $T_{4}(N, d)$ are internally disjoint.

We finally assume that $N-d<x \leq N-1$. Let $[x]_{d}=y$ for some $0 \leq x<<$. The paths from 0 to $x$ in $T_{2}(N, d)$ and in $T_{4}(N, d)$ are $0 \stackrel{+d}{\Longrightarrow} x-d-y \stackrel{+1}{\Longrightarrow} x-d \stackrel{+d}{\longrightarrow} x$ and $0 \stackrel{-d}{\longrightarrow} N-d \stackrel{+1}{\Longrightarrow} x$, respectively. These two paths are internally disjoint. Therefore, $T_{2}(N, d)$ and $T_{4}(N, d)$ are independent spanning trees rooted at 0 of $C R(N, d)$.

Lemma $7 T_{1}(N, d)$ and $T_{4}(N, d)$ are independent spanning trees rooted at 0 of $C R(N, d)$.

Proof: We first assume that $1 \leq x \leq d-1$. Let $[N-x]_{d}=y$ for some $0 \leq y<d$. The paths from 0 to $x$ in $T_{1}(N, d)$ and in $T_{4}(N, d)$ are $0 \stackrel{+1}{\Longrightarrow} x$ and $0 \stackrel{-d}{\Longrightarrow} x+d+y \stackrel{-1}{\Longrightarrow} x+d \stackrel{-d}{\longrightarrow} x$, respectively. These two paths are internally disjoint.

We next assume that $d \leq x \leq N-d$. If $[x]_{d}=0$ then the path from 0 to $x$ in $T_{1}(N, d)$ is $0 \stackrel{+1}{\Longrightarrow} d-1 \stackrel{+d}{\Longrightarrow} x-1 \stackrel{+1}{\longrightarrow} x$, and if $[x]_{d}=y$ for some $0<y<d$ then the path from 0 to $x$ in $T_{1}(N, d)$ is $0 \stackrel{+1}{\longrightarrow} y \stackrel{+d}{\longrightarrow} x$. For each case any internal vertex $a$ of the path satisfies $0<a<x$. As for the path from 0 to $x$ in $T_{4}(N, d)$, if $[N-x]_{d}=0$ then it is $0 \Longrightarrow x$, and if $[N-x]_{d}=y$ for some $0<y<d$ then it is $0 \stackrel{-d}{\Longrightarrow} x+y \stackrel{-1}{\Longrightarrow} x$. For each case any internal vertex $b$ satisfies $x<b \leq N-1$. Hence, for any case the paths from 0 to $x$ in $T_{1}(N, d)$ and $T_{4}(N, d)$ are internally disjoint.

We finally assume that $N-d<x \leq N-1$. The paths from 0 to $x$ in $T_{1}(N, d)$ and in $T_{4}(N, d)$ are $0 \stackrel{+1}{\longrightarrow}[x+d]_{N} \stackrel{-d}{\longrightarrow} x$ and $0 \stackrel{-d}{\longrightarrow} N-d \stackrel{+1}{\longrightarrow} x$, respectively. These two paths are internally disjoint. Therefore, $T_{1}(N, d)$ and $T_{4}(N, d)$ are independent spanning trees rooted at 0 of $C R(N, d)$. 
Symmetrically, we have the next lemma.

Lemma $8 T_{2}(N, d)$ and $T_{3}(N, d)$ are independent spanning trees rooted at 0 of $C R(N, d)$.

From Lemma 3 - Lemma 8, we have the next theorem.

Theorem 1 For any $2 \leq d<N / 2, T_{1}(N, d), T_{2}(N, d), T_{3}(N, d)$ and $T_{4}(N, d)$ are independent spanning trees rooted at 0 of $C R(N, d)$.

We next consider the case where $2 \leq d=N / 2$. In this case $C R(N, d)$ is 3 -comnected.

procedure construct $5(N, d)$;

$(* 2 \leq d=N / 2 *)$

begin

(1) connect vertex 0 with vertex $d$;

(2) for $i:=d$ to $N-2$ do connect vertex $i$ with vertex $i+1$;

(3) for $i:=d$ downto 2 do connect vertex $i$ with vertex $i-1$

end

Let $T_{5}(N, d)$ be a graph with the vertex set $\{0,1, \cdots, N-1\}$ and the edge set specified by procedure construct $5(N, d)$. We can show that $T_{5}(N, d)$ is a spanning tree rooted at 0 of $C R(N, d)$. For the case where $2 \leq d=N / 2$, we can also apply procedure construct $1(N, d)$. For this case we denote the spanning trees specified by procedure construct $1(N, d)$ by $T_{1}^{\prime}(N, d)$. Let $T_{3}^{\prime}(N, d)$ be a spanning tree of $C R(N, d)$ obtained from $T_{1}^{\prime}(N, d)$ by replacing the name of each vertex $x$ by $[N-x]_{N}$. In a similar way we can show the next theorem.

Theorem 2 For any $2 \leq d=N / 2, T_{1}^{\prime}(N, d), T_{3}^{\prime}(N, d)$ and $T_{5}(N, d)$ are independent spanning trees rooted at 0 of $C R(N, d)$.

In Figure 6 we show three independent spanning trees $T_{1}^{\prime}(14,7), T_{3}^{\prime}(14,7)$ and $T_{5}(14,7)$ of $C R(14,7)$.
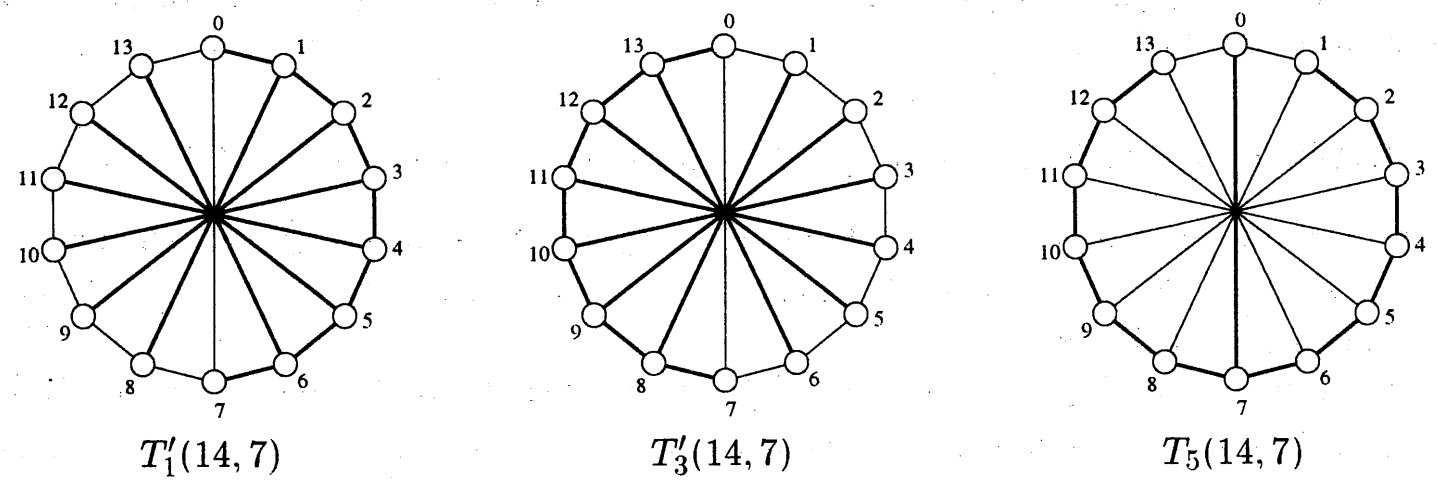

Figure 6: Three independent spanning trees of $C R(14,7)$

From Theorem 1 and Theorem 2 the following theorem is immediate.

Theorem 3 If $2 \leq d<N / 2, C R(N, d)$ is a 4-channel graph, and if $2 \leq d=N / 2, C R(N, d)$ is a 3-channel graph. 


\section{Concluding Remarks}

We can consider a more general family of chordal rings. A chordal ring $C R\left(N, d_{1}, \cdots, d_{k}\right)$ is a graph $G=(V, E)$, where $V=\{0,1, \cdots, N-1\}$ and $E=\left\{(u, v) \mid[v-u]_{N}=1\right.$ or $[v-u]_{N}=$ $d_{i}$ for some $\left.i(1 \leq i \leq k)\right\}$. Chordal rings discussed in this paper are the case where $k=1$ in the definition of the generalized chordal rings. In general we do not know how to construct efficiently the maximum number of independent spanning trees rooted at the same vertex of a generalized chordal ring. A graph $G=(V, E)$ with $N$ vertices is called the $N$-hyper-ring if $V=\{0,1, \cdots, N-1\}$ and $E=\left\{(u, v) \mid[v-u]_{N}\right.$ is a power of 2$\}$. Hyper-rings belong to the family of generalized chordal rings, and the network family has been much studied [1]. However, we do not know at present whether for each $N$, there are as many independent spanning trees rooted at the same vertex of $N$-hyper-ring as its connectivity.

It is also interesting to study how we design efficient broadcasting protocols for $C R(N, d)$, in particular for one-port mode, based on message transmission through independent spanning trees $T_{1}(N, d), T_{2}(N, d), T_{3}(N, d)$ and $T_{4}(N, d)$ (or $T_{1}^{\prime}(N, d), T_{3}^{\prime}(N, d)$ and $T_{5}(N, d)$ ).

These problems would be worthy for further investigation.

\section{References}

[1] T. Altman, Y. Igarashi, and K. Obokata, "Hyper-ring connection machine, Parallel Computing", vol.21, pp.1327-1338, 1995.

[2] F. Bao and Y. Igarashi, "Reliable broadcasting in product networks with Byzantine faults", 26th Annual International Symposium on Fault-Tolerant Computing, Sendai, Japan, pp.262-271, 1996.

[3] F. Bao, Y. Igarashi, and K. Katano, "Broadcasting in hypercubes with randomly distributed Byzantine faults", 9th International Workshop on Distributed Algorithms, Le Mont-Saint-Michel, France, LNCS vol.972, pp.215-229, 1995.

[4] F. Bao, Y. Igarashi, and S. R. Öhring, "Reliable broadcasting in product networks", IEICE Technical Report COMP 95-18, pp.57-66, 1995.

[5] G. D. Battista, R. Tamassia, and L. Vismara, "Output-sensitive reporting of disjoint paths", manuscript, Department of Computer Science, Brown University, 1996.

[6] J. Cheriyan and S. N. Maheshwari, "Finding nonseparating induced cycles and independent spanning trees in 3-connected graphs", J. Algorithms vol.9, pp.507-537, 1988.

[7] A. Huck, "Independent trees in graphs", Graphs Combin, vol.10, pp.29-45, 1994.

[8] A. Huck, "Independent trees in planar graphs", manuscript, Institut für Mathematik, Universität Hannover, 1996.

[9] A. Itai and M. Rodeh, "The multi-tree approach to reliability in distributed networks", Information and Computation, vol.79, pp.43-59, 1988.

[10] S. Khuller and B. Schieber, "On independent spanning trees", Information Processing Letters, vol.42, pp.321-323, 1992.

[11] K. Obokata, Y. Iwasaki, F. Bao, and Y. Igarashi, "Independent spanning trees of product, graphs", 22nd International Workshop on Graph-Theoretic Concepts in Computer Science, Como, Italy, 1996.

[12] A. Zehavi and A. Itai, "Three tree-paths", J. Graph Theory, vol.13, pp.175-188, 1989. 